\title{
Second-line treatment with nivolumab, cabozantinib, regorafenib, or best supportive care in patients with advanced hepatocellular carcinoma: analysis at a Hispanic-majority $\mathrm{NCl}-d e s i g n a t e d$ cancer center
}

\author{
Jeffrey Xia^^, Jonathan Gelfond, Sukeshi Patel Arora^ \\ Mays Cancer Center, University of Texas Health San Antonio, San Antonio, TX, USA \\ Contributions: (I) Conception and design: SP Arora, J Xia; (II) Administrative support: SP Arora; (III) Provision of study materials or patients: \\ SP Arora; (IV) Collection and assembly of data: J Xia, SP Arora; (V) Data analysis and interpretation: All authors; (VI) Manuscript writing: \\ All authors; (VII) Final approval of manuscript: All authors. \\ Correspondence to: Sukeshi Patel Arora, MD, Associate Professor, Leader Gastrointestinal Malignancies. Mays Cancer Center at UT Health \\ San Antonio, San Antonio, TX 78229, USA. Email: aroras@uthscsa.edu.
}

\begin{abstract}
Background: In the last four years, six regimens were approved by the Food and Drug Association as second-line therapies for advanced hepatocellular carcinoma (HCC). However, there are significant differences between real-world and clinical trial populations. We analyzed survival and toxicities among second-line therapies for HCC in our population.

Methods: We performed a retrospective cohort study of patients with advanced HCC who received second-line therapies (tyrosine kinase inhibitor or TKI; immunotherapy or IO) or best supportive care (BSC) at a tertiary-referral cancer center serving the South Texas region. Progression-free survival (PFS) was determined, and adverse events were compared between therapies.

Results: In our cohort, median age was 60 years $(n=65)$, and $49(75 \%)$ were Hispanic. $58(89 \%)$ patients received second-line therapy. Child-Pugh (CP) score of cohort: A, 18\%; B, 55\%; C, 26\%. Median PFS (mPFS) was 3.1 months with TKI ( $n=6), 3.3$ months with IO ( $n=27)$, and 1.3 months with BSC $(n=25)$. There was improved survival with IO compared to BSC [hazards ratio (HR) $=0.31 ; 95 \%$ confidence interval (CI): 0.15-0.63; $\mathrm{P}=0.0014]$. There was no significant difference comparing IO to TKI (HR $=0.94 ; 95 \%$ CI: 0.31-2.86; $\mathrm{P}=0.92)$, but a trend to improved PFS with TKI when compared to BSC (HR $=0.33 ; 95 \% \mathrm{CI}$ : 0.10-1.04; $\mathrm{P}=0.058)$. TKI group had significantly more rash $(\mathrm{P}=0.01)$ and hand-foot syndrome $(\mathrm{P}<0.001)$ compared to IO and BSC.

Conclusions: Our Hispanic-majority cohort with varying liver dysfunction, including CP-B \& C cirrhosis, were more likely to receive IO or BSC. Both second-line treatment groups, IO or TKI, demonstrated increased mPFS compared to BSC and were tolerable compared to BSC, with expected toxicity per class of drug.
\end{abstract}

Keywords: Real-world; survival; toxicity; liver; Hispanic

Submitted Jul 20, 2021. Accepted for publication Nov 03, 2021.

doi: 10.21037/jgo-21-414

View this article at: https://dx.doi.org/10.21037/jgo-21-414

^ ORCID: Jeffrey Xia, 0000-0002-2437-9954; Sukeshi Patel Arora, 0000-0002-1176-0713. 


\section{Introduction}

Hepatocellular carcinoma (HCC) is the fourth most common cause of cancer-related death and the seventh highest incident cancer internationally (1). The incidence of HCC has more than tripled within the last 30 years and continues to increase (2). Furthermore, the five-year survival rate of HCC is low, ranging from 33\% (early stage) down to $2 \%$ (distant metastasis) (2). Patients with advanced HCC unamenable to locoregional therapies or with progression on first-line systemic therapy face especially low survivability. Currently, these patients may elect to receive second-line systemic therapies.

Within the last four years, the FDA approved six regimens as second-line therapies for advanced HCC. These therapies include immunotherapies (IOs), vascular endothelial growth factor (VEGF) receptor inhibitors, and tyrosine kinase inhibitors (TKIs) targeting various aspects of the hallmarks of cancer (3).

Provided favorable treatment candidacy, patients with advanced HCC may elect for treatment with these second-line therapies after cancer progression or toxicity of first-line therapies, sorafenib and lenvatinib. However, it is important to understand that in clinical trials, strict inclusion criteria and protocols are employed to control for the intended variables of study. Trial populations often poorly represent real-world populations. One such example is the disproportionately higher inclusion of patients whose liver dysfunction is mild to moderate to determine treatment effect more accurately versus the natural disease course of advanced HCC. For example, only patients with Child-Pugh (CP)-A cirrhosis were included in the REACH-2 (ramucirumab), KEYNOTE-224 (pembrolizumab), and RESORCE (regorafenib) trials (4-6). The CELESTIAL (cabozantinib) and Checkmate-040 (nivolumab) trials extended selection criteria to include patients up to CP-B7 and/or B8 cirrhosis but not B9 $(7,8)$. Furthermore, the etiology of HCC often significantly differs between trial studies and real-world populations, and in particular the United States. For example, the percent prevalence of hepatitis B virus (HBV) in the clinical trials ranged between $21-51 \%$, while the prevalence of HCC secondary to HBV in the US is lower at $10-15 \%$ (9). The percent prevalence of hepatitis $\mathrm{C}$ virus (HCV) in the trials ranged between $21-29 \%$, while the prevalence of HCC secondary to HCV in the US is higher at $50-60 \%$ (9). Lastly, many of these clinical trials were conducted as international, multi-center studies, enrolling patient pools that widely differ in regional demographics. In the trials, people of Hispanic ethnicity are categorized as White race, and the percentage of White subjects ranged between $20-81 \%$ in the trial studies. Thus, it is unclear of the level of representation for Hispanics in the trials, though it is likely Hispanics are underrepresented.

Real-world application of clinical trials remains a challenge due to a heterogeneous population of varying liver dysfunction, ethnicities, and cirrhosis etiology, signaling for the need of more investigation of second-line therapies in such diverse and unique populations. At our cancer center, the majority of our patients are Hispanic with varying liver dysfunction, and this real-world population is not represented in registration trials of TKIs and IOs. Therefore, we analyzed survival and toxicities among second-line therapies for HCC at our Hispanic-majority NCI-designated cancer center. We present the following article in accordance with the STROBE reporting checklist (available at https://dx.doi.org/10.21037/jgo-21-414).

\section{Methods}

We conducted a retrospective cohort analysis of patients diagnosed with advanced HCC who received standard of care first-line systemic therapy, sorafenib or lenvatinib, and second-line systemic therapy at our tertiary referral center in South Texas from 2015-2019. Of the five FDAapproved second-line therapies, as a result of physician's choice for treatment based on National Comprehensive Cancer Network (NCCN) guidelines, patients received nivolumab (IO), regorafenib (TKI), or cabozantinib (TKI) at our center during the time frame of this retrospective study (Figure 1) (10). Patients who were not candidates for second-line therapies received best supportive care (BSC). Two patients were enrolled in clinical trials after failure of first-line systemic therapy and were not included in this analysis. Baseline liver function studies were collected upon initiation of second-line therapy to determine CP class, Barcelona Clinic Liver Cancer (BCLC) stage, and Model for End-Stage Liver Disease (MELD) score. Patients were assessed at four-week intervals with history, physical exam, and laboratory evaluation. Any dose reductions or interruptions were documented during visits.

Nivolumab was administered at a dose of $480 \mathrm{mg}$ intravenously every 4 weeks. Regorafenib was administered at a dose of $160 \mathrm{mg}$ orally daily, 3 weeks on and 1 week off. At the discretion of the oncologist, some patients were started at $80 \mathrm{mg}$ daily due to frailty, advanced age, or 


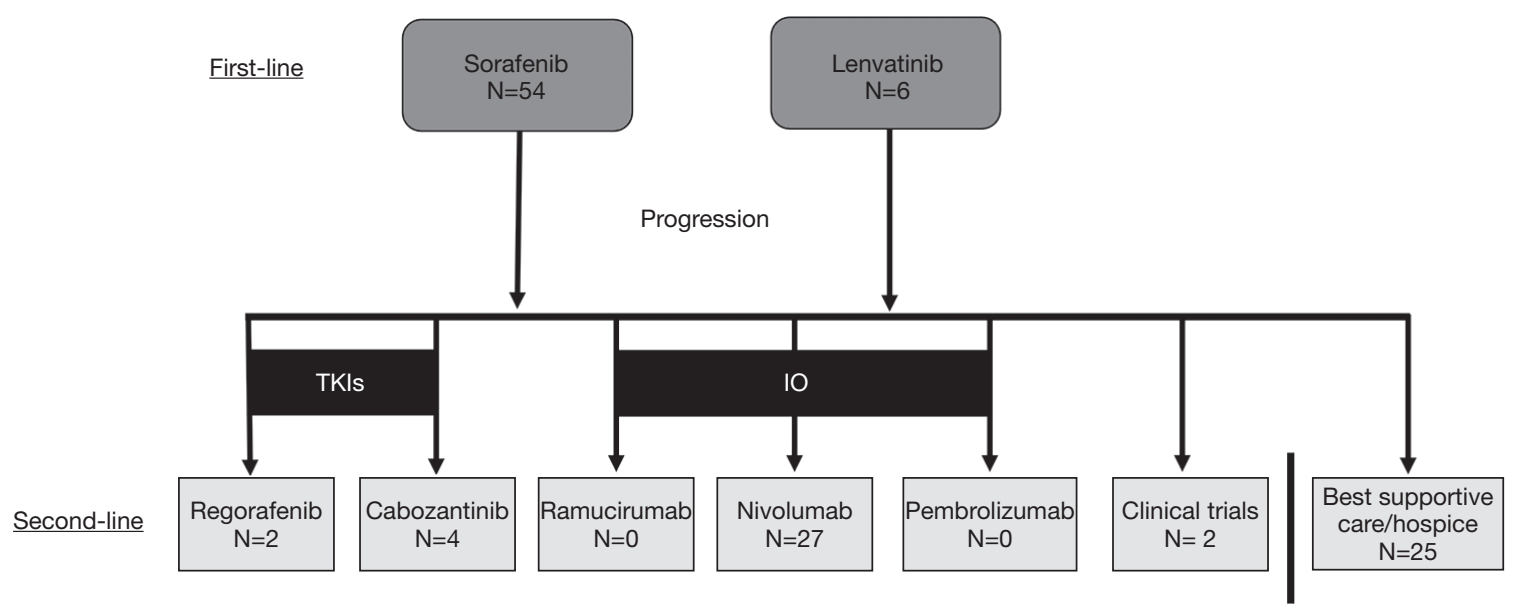

Figure 1 Treatment algorithm and patient groups for advanced cellular carcinoma treated at Mays Cancer Center from January 2015 to March 2019. TKI, tyrosine kinase inhibitor; IO, immunotherapy.

significant liver dysfunction, with the goal of titrating up to $160 \mathrm{mg}$ daily. Cabozantinib was administered at a dose of $40 \mathrm{mg}$, titrated up to $60 \mathrm{mg}$ as tolerated. Treatment was continued until disease progression, unacceptable toxicity, lost to follow-up or withdrawal of patient consent. Documentation of disease progression was the primary endpoint for patients who were not candidates of secondline therapy and received BSC or hospice.

Treatment response of the second-line therapies were assessed clinically and radiographically by oncologists and radiologists typically completed every 12 weeks. Physicians relied upon radiologist's reading of the CT or MRI compared to baseline.

Adverse events (AEs) were documented upon discovery during interval history, physical exam, labs, or other lines of communication with provider. AEs were graded according to the NCI Common Terminology Criteria for Adverse Events version 5.0.

\section{Statistical analysis}

The association between demographic, clinical, and laboratory variables with progression-free survival (PFS) were assessed by Cox proportional hazards models and comparison of Kaplan-Meier curves. Groups were statistically compared by the log rank test, and the magnitude of association with outcome was summarized as the hazards ratio (HR) with $95 \%$ confidence interval (CI). The $\mathrm{R}$ statistical computing environment (version 4.0, Vienna, Austria) was used for all statistical analyses.

\section{Ethical statement}

The study was conducted in accordance with the Declaration of Helsinki (as revised in 2013). The IRB of University of Texas Health at San Antonio has reviewed this research and granted approval for meeting requirements of protection of human subjects (No. HSC20190229E). Per IRB, informed consent was not required due to the retrospective nature of this study.

\section{Results}

\section{Demographics}

Sixty-five patients received first-line systemic therapy for advanced HCC. Out of the 65 patients, 58 (89\%) patients received second-line therapy or BSC due to failure of first-line therapy. The median age of the cohort was 60 years $(n=65)$. Analysis of demographics in this cohort was performed by separating the cohort into treatment groups (BSC, IO, TKI) (Table 1). Hispanic ethnicity was the most common ethnicity in this cohort, with the following cohort distribution: $75 \%(\mathrm{n}=49)$ were Hispanic White, $18 \%(\mathrm{n}=12)$ were non-Hispanic White, and 6\% $(n=4)$ were Black. Male sex was predominant at $89 \%(\mathrm{n}=58)$. HCV infection was the most common cause of HCC $(74 \%, n=48)$, followed by alcoholic cirrhosis $(60 \%, \mathrm{n}=39)$, and fatty liver disease $(20 \%$, $\mathrm{n}=13)$. Most patients had CP-B cirrhosis, with the following cohort distribution: 18\% CP-A, 55\% CP-B (B7, 21.5\%; B8, $21.5 \%$; B9, $12.3 \%$ ), and 26\% CP-C. Most patients had BCLC stage C HCC, with the following cohort 
Table 1 Demographic data and baseline characteristics of liver dysfunction in a cohort of patients with advanced HCC after failure of first-line therapy treated at Mays Cancer Center

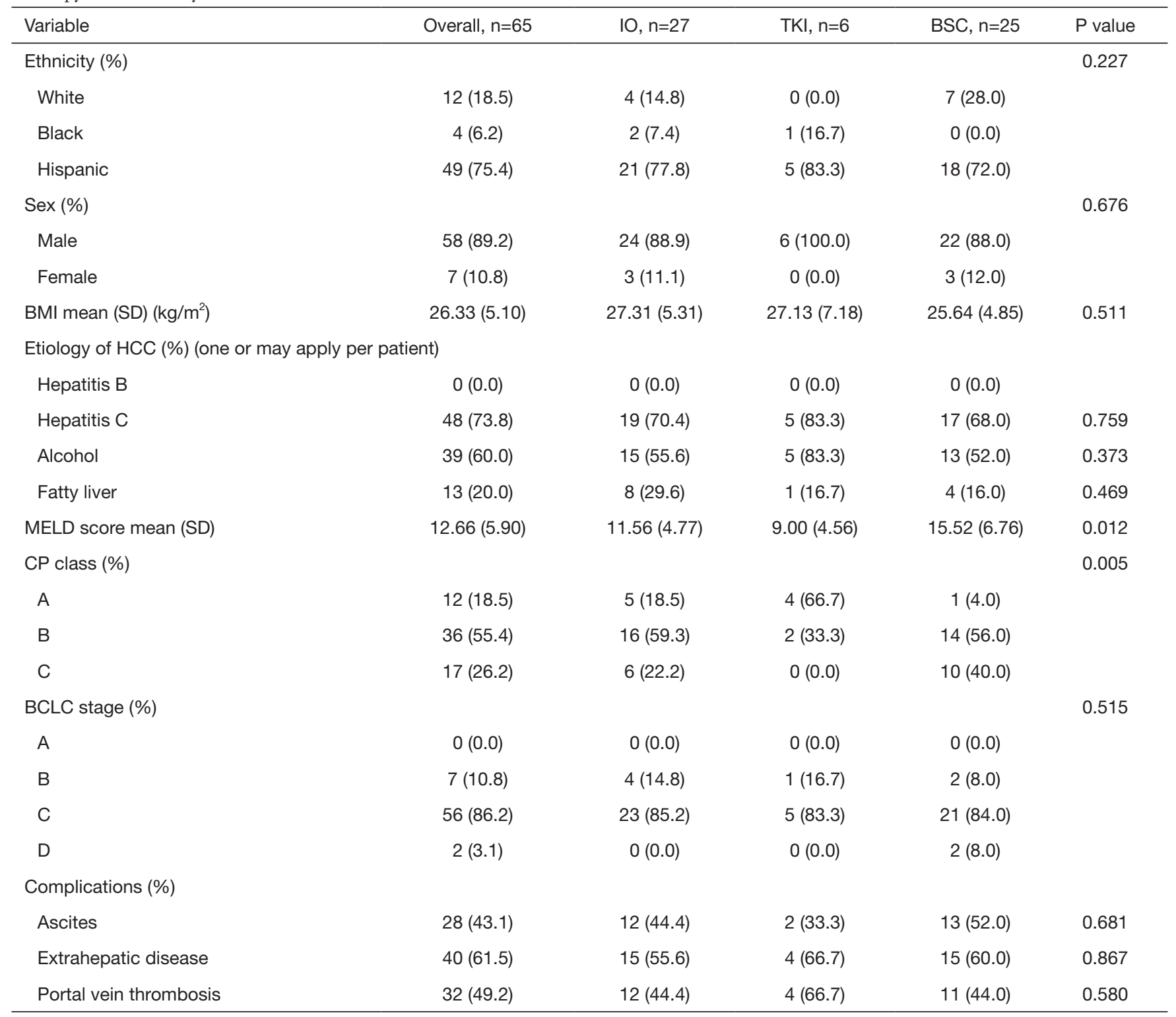

HCC, hepatocellular carcinoma; IO, immunotherapy (nivolumab); TKI, tyrosine kinase inhibitor (regorafenib, cabozantinib); BSC, best supportive care; MELD, Model for End-Stage Liver Disease; CP, Child-Pugh; BCLC, Barcelona Clinic Liver Cancer.

distribution: $0 \%$ stage A; $11 \%(n=7)$ stage $B ; 86 \%(n=56)$ stage $C$; and $3 \%(n=2)$ stage $D$. Additionally, $43 \%(n=28)$ of patients had ascites, $62 \%(\mathrm{n}=40)$ had extrahepatic disease, and $49 \%(n=32)$ had portal vein thrombosis.

\section{Sex and etbnicity}

In our cohort, male sex demonstrated significantly decreased survival compared to females ( $\mathrm{HR}=4.73 ; 95 \% \mathrm{CI}$ :
1.32-16.97; $\mathrm{P}=0.017)$. There was no significant difference in survival between Hispanic and non-Hispanic White ethnicities (HR $=0.82 ; 95 \%$ CI: $0.35-1.89 ; \mathrm{P}=0.637$ ), nor between Black and non-Hispanic White ethnicities (HR $=1.74 ; 95 \%$ CI: $0.28-10.75 ; \mathrm{P}=0.550$ ).

\section{Age}

In comparison of older ( $\geq 60$ years) and younger adults 
Table 2 Univariate Cox regression analysis for median progression free survival stratified by second-line therapeutic category in patients with advanced HCC

\begin{tabular}{lcccc}
\hline Treatment & $\begin{array}{c}\text { mPFS } \\
\text { (months) }\end{array}$ & HR & $95 \% \mathrm{Cl}$ & P value \\
\hline BSC & 1.3 & - & - & - \\
IO & 3.3 & 0.31 & $(0.15-0.63)$ & 0.0014 \\
TKI & 3.1 & 0.33 & $(0.10-1.04)$ & 0.058 \\
\hline
\end{tabular}

HCC, hepatocellular carcinoma; mPFS, median progression free survival; HR, hazard ratio; $\mathrm{Cl}$, confidence interval; $\mathrm{BSC}$, best supportive care; IO, immunotherapy (nivolumab); TKI, tyrosine kinase inhibitor (cabozantinib, regorafenib).

( $<60$ years) in our cohort, younger adults had significantly greater prevalence $(\mathrm{P}=0.013)$ of $\mathrm{HCV}$ cirrhosis. Average MELD score was 12.71 and 12.61 for the younger and older group, respectively. There was no significant difference in survival between younger and older groups ( $\mathrm{HR}=0.78 ; 95 \%$ CI: $0.41-1.49$; $\mathrm{P}=0.449$ ).

\section{Efficacy}

Of the 58 patients who received second-line therapy, the median PFS (mPFS) was 3.1 months with TKI $(n=6)$, 3.3 months with IO $(\mathrm{n}=27)$, and 1.3 months with BSC $(\mathrm{n}=25)$ (Table 2). There was improved survival with IO when compared to BSC (HR $=0.31 ; 95 \%$ CI: 0.15-0.63; $\mathrm{P}=0.0014)$. There was no significant difference when comparing IO to TKI (HR $=0.94$; 95\% CI: 0.31-2.86; $\mathrm{P}=0.92$ ), but a trend to improved PFS with TKI when compared to BSC (HR $=0.33 ; 95 \%$ CI: 0.10-1.04; $\mathrm{P}=0.058$ ).

\section{Toxicity}

There was significantly more rash $(\mathrm{P}=0.01)$ and handfoot syndrome $(\mathrm{P}<0.001)$ in the TKI group compared to the IO and BSC groups (Table 3). There was no significant difference between groups for all other adverse events including nausea, fatigue, diarrhea, immune-mediated reactions, cytopenias, and elevated blood pressure. Of the entire cohort, $38 \%(\mathrm{n}=25)$ of the cohort experienced nausea, $78 \%(\mathrm{n}=51)$ experienced fatigue, $18 \%(\mathrm{n}=12)$ experienced diarrhea, $6 \%(\mathrm{n}=4)$ experienced hand-foot syndrome, $20 \%$ $(\mathrm{n}=13)$ experienced rashes excluding hand-foot syndrome, $14 \%(\mathrm{n}=9)$ experienced immune mediated reactions, and $6 \%$ $(\mathrm{n}=4)$ experienced cytopenias. All patients who had handfoot syndrome $(n=4)$ were in the TKI group, and most patients who experienced immune-mediated reactions $(\mathrm{n}=8)$ were in the IO group.

\section{Discussion}

In this study, we investigated the real-world efficacy and toxicity of second-line therapies in patients with advanced HCC after first-line systemic therapy at our South Texas tertiary referral cancer center. In our Hispanic-majority cohort, patients with varying liver dysfunction, including CP-B and CP-C cirrhosis, were more likely to receive IO or BSC as second-line systemic therapy, respectively. Both treatment groups (IO or TKI) demonstrated increased mPFS compared to BSC (Figure 2). Both IO and TKI groups were tolerable compared to BSC, with expected toxicity per class of drug.

Regarding IO, in the CheckMate-040 trial, the mPFS for nivolumab was 3.4 months (95\% CI: 1.6-6.9), which is comparable to our cohort's mPFS of 3.3 months (95\% CI: 3.0-7.0) (8). This may be due to more comparable racial demographics and inclusion of patients with CP-B liver dysfunction; however, our study did include CP-B9, which were excluded from CheckMate-040. The CheckMate-040 trial was $49 \%$ White and $2 \%$ CP-B, while our cohort was $93 \%$ White with $55 \%$ CP-B (8). Regarding TKIs, in the CELESTIAL and RESORCE trials, the mPFS for cabozantinib and regorafenib were 5.2 (95\% CI: 4.0-5.5) and 3.1 (95\% CI: 2.8-4.2) months, respectively $(6,7)$. Our collective TKI group demonstrated a mPFS of 3.1 months (95\% CI: $1.2-\infty)$. The CELESTIAL trial cohort was $58 \%$ White, and no CP-B or CP-C cirrhosis were enrolled at diagnosis, however, 51 patients in the cabozantinib arm (11\%) had evolved to CP-B cirrhosis at 8 weeks (11). This cohort of patients had a mPFS of 3.7 versus 1.9 months (HR $=0.44$; 95\% CI: 0.25-0.76) (10). The RESORCE trial cohort included 36\% White with predominance of Asian race and $1.9 \% \mathrm{CP}-\mathrm{B}$ cirrhosis with no $\mathrm{CP}-\mathrm{C}$ cirrhosis (4). Based on these findings, registration trials which included even a small number of patients with more advanced liver dysfunction (CP-B or greater) showed more comparable efficacy with our real-world cohort consisting of $81 \% \mathrm{CP}-\mathrm{B}$ and $\mathrm{C}$ liver dysfunction. From these results, it is more evident that lack of inclusion of patients with advanced cirrhosis such as in the CELESTIAL trial resulted in a greater difference between trial and real-world second-line treatment efficacy.

Our cohort has unique characteristics compared to trial cohorts, with a majority of Hispanic ethnicity and 
Table 3 Adverse effects and toxicity assessed by the NCI Common Terminology Criteria for Adverse Events version 5.0 for patients with advanced HCC treated with IO, TKI, and BSC

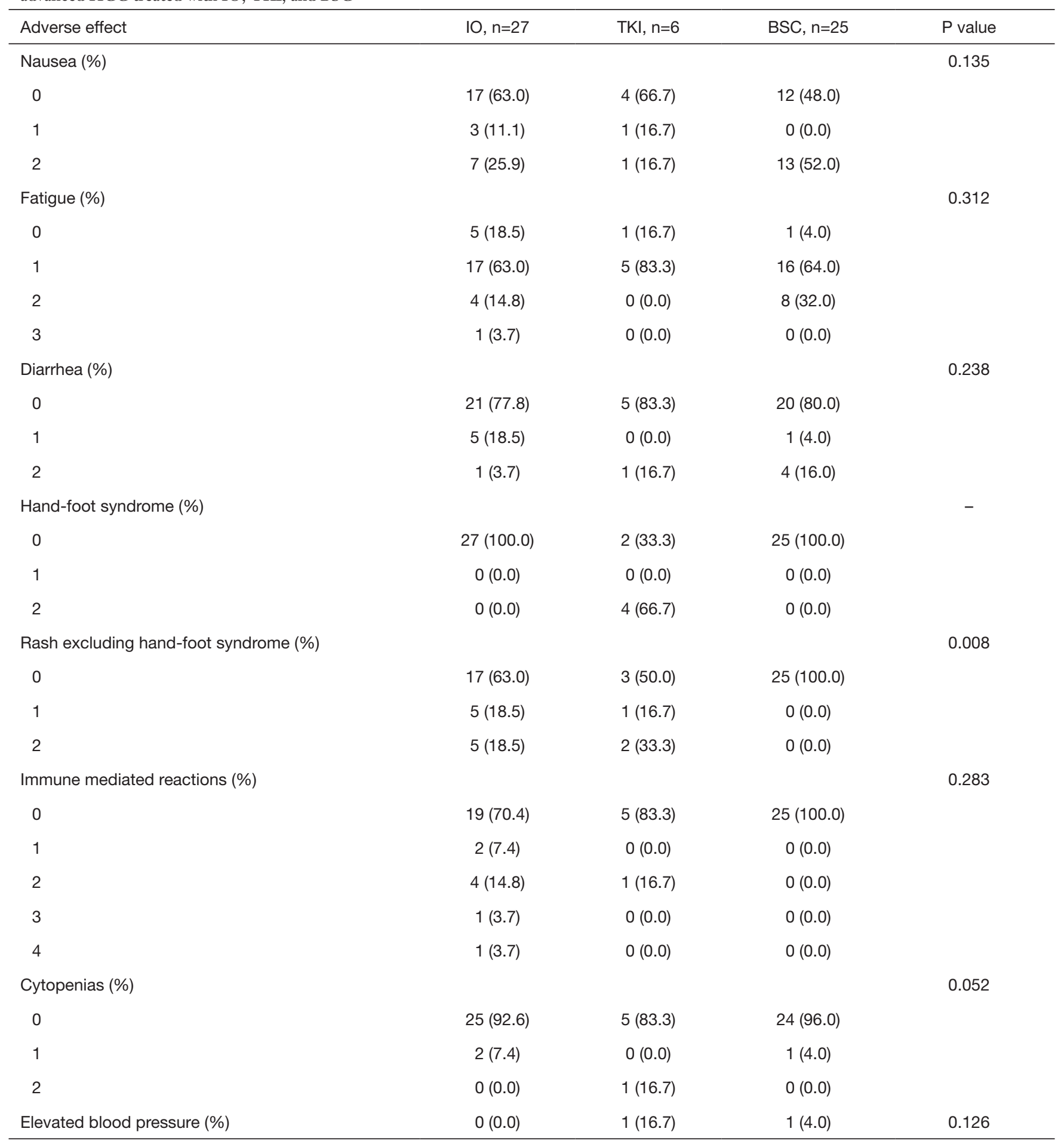

HCC, hepatocellular carcinoma; IO, immunotherapy; TKI, tyrosine kinase inhibitor; BSC, best supportive care. 


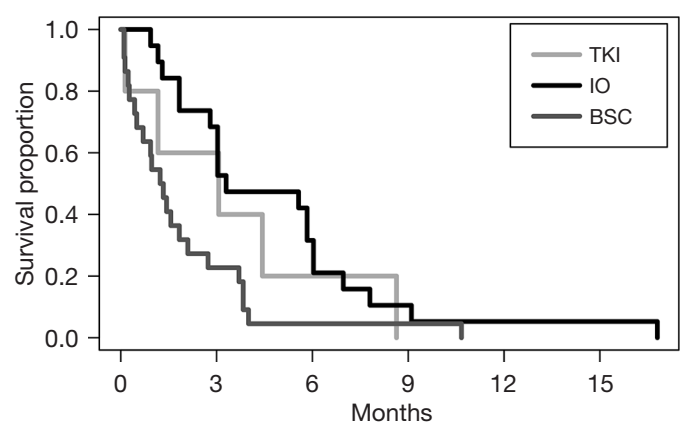

Figure 2 Kaplan-Meier survival curves for each second-line therapy group and BSC for patients with advanced HCC. TKI, tyrosine kinase inhibitor; IO, immunotherapy; BSC, best supportive care; HCC, hepatocellular carcinoma.

etiology of HCC primarily due to HCV and alcoholic cirrhosis with minimal HBV cirrhosis. Other retrospective cohort analyses have shown variable survival depending on the cohort demographics. In a study investigating second-line therapies for HCC in Canada, median overall survival of cabozantinib and regorafenib were 8.8 and 9.7 months, respectively (12). While this cohort was more heterogenous and contained higher variance in liver dysfunction compared to trial studies, this cohort does have significantly more $\mathrm{HBV}(32.2 \%)$ and less alcoholic cirrhosis $(22.1 \%)$ compared to our cohort, which may explain the difference in mPFS (12). In a US study with a majority $\mathrm{HCV}$ cohort (56.1\%) comparing second-line IO to non-IO, the mPFS for the groups were 3.9 and 3.0 months, respectively (12). In this study, there was no significant difference in mPFS between IO and non-IO treatment groups, but the survival curves depict a delayed survival effect for the IO group (13). These results were similar to our results, which may be due to the comparable HCV prevalence between cohorts. The mPFS for the IO group in our study appears to be shorter; the reason is likely multifactorial, which may include differences in cohort ethnicity and that pembrolizumab, a checkpoint inhibitor of related mechanism to nivolumab, was included in their IO category. In another study comparing regorafenib (TKI) and nivolumab (IO) in Korea, mPFS of regorafenib and nivolumab were 5.4 and 8.9 months, respectively. Again, cohort differences in HCC etiology (79.8-83.3\% HBV) and ethnicity (majority Korean Asian) may explain the variability of mPFS, yet this study also included greater variance in liver dysfunction expected in real-world studies. Furthermore, this study reflected our findings of no significant difference in survival between nivolumab (IO) and regorafenib (TKI), though with slightly improved survival in the IO group (14). Two more studies conducted in Korea investigating regorafenib efficacy after sorafenib treatment failure demonstrated mPFS of 2.7 and 3.7 months in their cohorts with majority $\mathrm{HBV}$ cirrhosis and CP-A cirrhosis, which were comparable to our findings $(15,16)$.

One of the strengths of our study was the investigation of a patient population significantly different from trial studies. Our cohort comprises a unique patient population with a Hispanic majority. Furthermore, our study cohort included patients observed in a real-world setting, where studies are lacking for patients with liver dysfunction (CP-B and CP-C).

The limitations of our study include a small cohort size and a retrospective cohort study at a single institution. Retrospective cohort studies have inherent disadvantages such as a relatively weaker level of evidence compared to prospective cohort studies and clinical trials because treatment selection could be confounded with patient characteristics. In our study, second-line treatment was selected by physician's choice based on NCCN guidelines. As this is not a randomized study, but a retrospective analysis, the groups were not balanced in regards to $\mathrm{CP}$ status (Table 1). This IO group had more CP-B patients versus TKI group had more CP-A patients versus BSC had more CP-B and CP-C patients. This can possibly impact survival as well. Patients in each group had a similar stage for HCC, which was majority BCLC B: IO $85.2 \%$, TKI $83.3 \%$, BSC $84.0 \%$. Given this small cohort, a larger randomized study would address these biases.

However, clinical trials usually exclude patients with liver dysfunction, and there is a great unmet need for this patient population. Our study adds to the real-world data to provide evidence for clinical decision-making in the treatment of advanced HCC in underrepresented populations. Epidemiological studies revealed Hispanics have the highest rate of HCC in the United States. Furthermore, there is a statistically higher geographical incidence of HCC in the southern United States, with Texas having the highest HCC incidence in the nation (17). HCC incidence in Texas is $40 \%$ to $80 \%$ higher than national levels for age groups $45-54$ and 65-74 years, respectively (17). Due to the limited cohorts in the trial studies supporting second-line therapies, it is important to continue investigating the efficacy and safety of utilizing these agents in underrepresented ethnicities such as Hispanics.

Regarding future directions, as the FDA approves more 
second-line therapies for advanced HCC, more prospective studies are needed to compare efficacy and safety of these agents in patients with CP-B cirrhosis. Additionally, while treatment response for HCC is often assessed clinically and radiographically, there is a need for more reliable and sensitive biomarkers of efficacy to guide treatment choices for TKIs versus IO versus BSC. Lastly, patients with advanced HCC and CP-B cirrhosis need to be included in phase II and III studies as these patients are currently underrepresented due to exclusion from clinical trials.

\section{Acknowledgments}

Funding: This work was supported by the National Institutes of Health (grant number CA054174).

\section{Footnote}

Reporting Checklist: The authors have completed the STROBE reporting checklist. Available at https://dx.doi. org/10.21037/jgo-21-414

Data Sharing Statement: Available at https://dx.doi. org/10.21037/jgo-21-414

Peer Review File: Available at https://dx.doi.org/10.21037/ jgo-21-414

Conflicts of Interest: All authors have completed the ICMJE uniform disclosure form (available at https://dx.doi. org/10.21037/jgo-21-414). SPA reports that she is on the speaker's bureau for Exelixis, Bristol-Meyers Squibb, and Bayer and advisory board for AstraZeneca and QED Therapeutics, and SPA's institution has received research funding from Beigene, Faron, Halozyme, Ipsen, Lexicon, Lilly, and Caris Life Sciences, and received Travel support from Faron, outside the submitted work. The other authors have no conflicts of interest to declare.

Ethical Statement: The authors are accountable for all aspects of the work in ensuring that questions related to the accuracy or integrity of any part of the work are appropriately investigated and resolved. The study was conducted in accordance with the Declaration of Helsinki (as revised in 2013). The IRB of University of Texas Health San Antonio has reviewed this research and granted approval for meeting requirements of protection of human subjects (No. HSC20190229E). Per IRB, informed consent was not required due to the retrospective nature of this study.

Open Access Statement: This is an Open Access article distributed in accordance with the Creative Commons Attribution-NonCommercial-NoDerivs 4.0 International License (CC BY-NC-ND 4.0), which permits the noncommercial replication and distribution of the article with the strict proviso that no changes or edits are made and the original work is properly cited (including links to both the formal publication through the relevant DOI and the license). See: https://creativecommons.org/licenses/by-nc-nd/4.0/.

\section{References}

1. Sung H, Ferlay J, Siegel RL, et al. Global Cancer Statistics 2020: GLOBOCAN Estimates of Incidence and Mortality Worldwide for 36 Cancers in 185 Countries. CA Cancer J Clin 2021;71:209-49.

2. Siegel RL, Miller KD, Jemal A. Cancer statistics, 2020. CA Cancer J Clin 2020;70:7-30.

3. Hanahan D, Weinberg RA. Hallmarks of cancer: the next generation. Cell 2011;144:646-74.

4. Zhu AX, Kang YK, Yen CJ, et al. Ramucirumab after sorafenib in patients with advanced hepatocellular carcinoma and increased $\alpha$-fetoprotein concentrations (REACH-2): a non-randomized, double-blind, placebocontrolled, phase 3 trial. Lancet Oncol 2019;20:282-96.

5. Zhu AX, Finn RS, Edeline J, et al. Pembrolizumab in patients with advanced hepatocellular carcinoma previously treated with sorafenib (KEYNOTE-224): a non-randomised, open-label phase 2 trial. Lancet Oncol 2018;19:940-52.

6. Bruix J, Qin S, Merle P, et al. Regorafenib for patients with hepatocellular carcinoma who progressed on sorafenib treatment (RESORCE): a randomized, double-blind, placebo-controlled, phase 3 trial. Lancet 2017;389:56-66.

7. Abou-Alfa GK, Meyer T, Cheng AL, et al. Cabozantinib in Patients with Advanced and Progressing Hepatocellular Carcinoma. N Engl J Med 2018;379:54-63.

8. El-Khoueiry AB, Sangro B, Yau T, et al. Nivolumab in patients with advanced hepatocellular carcinoma (CheckMate 040): an open-label, non-comparative, phase 1/2 dose escalation and expansion trial. Lancet 2017;389:2492-502.

9. El-Serag HB. Epidemiology of viral hepatitis and hepatocellular carcinoma. Gastroenterology 2012;142:1264-1273.e1.

10. National Comprehensive Cancer Network (NCCN) 
Guidelines: Hepatobiliary Cancers. Version 5.2021. Available online: https://www.nccn.org/professionals/ physician_gls/pdf/hepatobiliary.pdf

11. El-Khoueiry A, Meyer T, Cheng A, et al. SO-9 Outcomes for patients with advanced hepatocellular carcinoma and Child-Pugh B liver function in the phase 3 CELESTIAL study of cabozantinib vs placebo. Ann Oncol 2020;31:S220.

12. Fung AS, Tam VC, Meyers DE, et al. Second-line treatment of hepatocellular carcinoma after sorafenib: Characterizing treatments used over the past 10 years and real-world eligibility for cabozantinib, regorafenib, and ramucirumab. Cancer Med 2020;9:4640-7.

13. Saeed A, Hildebrand H, Park R, et al. Immune Checkpoint Inhibitors versus VEGF Targeted Therapy as Second Line Regimen in Advanced Hepatocellular Carcinoma (HCC): A Retrospective Study. J Clin Med 2020;9:2682.

14. Choi WM, Choi J, Lee D, et al. Regorafenib Versus

Cite this article as: Xia J, Gelfond J, Arora SP. Secondline treatment with nivolumab, cabozantinib, regorafenib, or best supportive care in patients with advanced hepatocellular carcinoma: analysis at a Hispanic-majority NCI-designated cancer center. J Gastrointest Oncol 2021;12(6):2943-2951. doi: 10.21037/ggo-21-414
Nivolumab After Sorafenib Failure: Real-World Data in Patients With Hepatocellular Carcinoma. Hepatol Commun 2020;4:1073-86.

15. Lee MJ, Chang SW, Kim JH, et al. Real-world systemic sequential therapy with sorafenib and regorafenib for advanced hepatocellular carcinoma: a multicenter retrospective study in Korea. Invest New Drugs 2021;39:260-8.

16. Yoo C, Park JW, Kim YJ, et al. Multicenter retrospective analysis of the safety and efficacy of regorafenib after progression on sorafenib in Korean patients with hepatocellular carcinoma. Invest New Drugs 2019;37:567-72.

17. El-Serag HB, Sardell R, Thrift AP, et al. Texas Has the Highest Hepatocellular Carcinoma Incidence Rates in the USA. Dig Dis Sci 2021;66:912-6. 